Recibido:

13-IX-2019

Aceptado:

7-X-2019

Publicado en línea: 18-X-2019
Osteoporosis en los maxilares y sus métodos de diagnóstico: Revisión de literatura

\section{Osteoporosis of the Jaws and its Diagnostic Methods:}

\section{Review of the Literature}

Jacqueline Jovana Rocha Claros Mg1

1. Posgraduante en la división de Radiología Oral y Maxilofacial, carrera de Odontología Universidad Científica del Sur-UCSUR, en coordinación con el Instituto Latino Americano de Altos Estudios ILAE y la División de Educación Continua del Centro de Diagnóstico por Imágenes. Lima-Perú. Docente de pregrado Facultad de Odontología, catedra de Radiología bucal, Universidad Mayor de San Simón. Cochabamba, Bolivia.

Autor para correspondencia: Dra. Jacqueline Jovana Rocha Claros - jrochaclaros@odo.umss.edu.bo

RESUMEN: La osteoporosis es una enfermedad sistémica y metabólica ósea, multifactorial, crónica, silenciosa, caracterizada por una baja densidad mineral ósea y alteración de su micro arquitectura, con un alto índice de fracturas, con trauma mínimo o sin trauma (cadera, columna vertebral y antebrazos). La osteoporosis se puede dar en ambos sexos y en todas las edades, considerándose principalmente un problema de salud femenino después de la menopausia, con riesgo de fracturas por encima de los 50 años. Esto aumenta la morbilidad, la mortalidad y los costos de atención médica, considerándose un problema significativo de salud pública. El hueso normal tiene características de equilibrio entre formación de hueso por los osteoblastos y la resorción de hueso por Ios osteoclastos. En la osteoporosis este equilibrio se altera y hay mayor tendencia a la resorción. Los factores de riesgo para la osteoporosis son varios, entre los que destacan la edad, sexo, factores genéticos, menopausia temprana, inadecuado consumo de calcio, falta de ejercicios, alcoholismo y tabaquismo; pueden estar asociado a ciertas enfermedades endocrinas como el hiperparatiroidismo, insuficiencia renal crónica, enfermedad hepática, malabsorción y fármacos como el glucocorticoide oral. El objetivo de la presente revisión bibliográfica es brindar información actualizada y específica sobre la osteoporosis en los maxilares y sus métodos de diagnóstico, que permitan al odontólogo y radiólogo, reconocer, prevenir y/o derivar al especialista indicado los casos que puedan presentarse con esta condición.

PALABRAS CLAVE: Osteoporosis; Radiografía panorámica; Densidad ósea. 
ABSTRACT: Osteoporosis is a chronic, silent, multifactorial and metabolic bone disease characterized by low bone mineral density and altered micro architecture, presenting a high fracture rate with minimal trauma or without trauma (hip, spine and forearms). Osteoporosis can occur in both sexes and in all ages; mainly considered a problem for postmenopausal women, with higher risk of fracture over 50 years old. This increases the morbidity, mortality and costs of the health system, considering it a significant public health issue. Healthy bone has equilibrium characteristics between bone formation by osteoblasts and bone resorption by osteoclasts. In osteoporosis this balance is altered, showing a greater tendency to resorption. The most important risk factors for osteoporosis are age, sex, genetic factors, early menopause, inadequate calcium intake, lack of exercise, alcoholism, and smoking; it can also be associated with some endocrine diseases, such as hyperparathyroidism, kidney failure chronic, liver disease, malabsorption and drugs, including oral glucocorticoids. The objective of this bibliographic review is to provide updated and specific information about osteoporosis in the jaws and its diagnostic methods, which allow the dentist and radiologist to recognize, prevent and / or refer to the specialist indicated cases that may arise with this condition.

KEYWORDS: Osteoporosis; Panoramic Radiography; Bone Density.

\section{INTRODUCCIÓN}

Laosteoporosis se define comouna enfermedad sistémica y metabólica ósea, caracterizada por una baja densidad mineral ósea, con alteración en la micro arquitectura del tejido óseo, lo cual genera una mayor susceptibilidad a la fractura. (OMS 1994) (1-5). Es una enfermedad multifactorial crónica, silenciosa, con un alto índice de fracturas, con trauma mínimo o sin trauma (cadera, columna vertebral y antebrazos). La osteoporosis se puede dar en ambos sexos y en todas las edades, siendo generalmente un problema de salud femenino después de la menopausia, con riesgo de fracturas por encima de los 50 años, incrementando la morbilidad, la mortalidad y los costos del sistema de salud $(6,7)$.

Las características del hueso sano se dan por el equilibrio entre formación de hueso por los osteoblastos y la reabsorción por los osteoclastos, en osteoporosis este equilibrio se altera y hay mayor tendencia a la reabsorción.
Los factores de riesgo para la osteoporosis son varios, entre los que destacan la edad, sexo, factores genéticos, menopausia temprana, inadecuado consumo de calcio, falta de ejercicios, alcoholismo y tabaquismo. Puede también estar asociados a ciertas enfermedades endocrinas como el hiperparatiroidismo, insuficiencia renal crónica, enfermedad hepática, malabsorción y fármacos como glucocorticoides orales $(8,9)$. La disminución de los niveles de estrógenos y de calcio en mujeres posmenopáusicas, se consideran como los factores principales de osteoporosis (10-12). Así también, otros factores de riesgo asociados a osteoporosis serían los niveles altos de homocisteína y una baja Lipoproteína de Alta Densidad (HDL) 0 colesterol bueno, que se relaciona con enfermedad cardiovascular y aumentaría con la edad en mujeres posmenopáusicas $(13,14)$.

Según la OMS 2003, aproximadamente 75 millones de personas en Europa, Japón y los Estados Unidos padecen este problema, causando más de 2.3 millones de fracturas al año, cantidades 
que en los próximos 50 años se duplicarán según los organismos internacionales, presagiando un aumento de osteoporosis tanto en Asia, como en América Latina, aumentando la morbilidad, la mortalidad y los costos del sistema de salud, considerándose un problema significativo de salud pública $(1,15)$. En los últimos años, estudios han determinado una relación entre la osteoporosis y la salud oral, principalmente con la enfermedad periodontal (16). La relación de pérdida de hueso de la cavidad bucal y la osteoporosis sería frecuente, sin embargo, aún se requieren estudios más concluyentes para inferir causalidad (17-19). Analizar el hueso mandibular permitirá predecir fracturas osteoporóticas, por el metabolismo óseo rápido de este hueso que es el doble que, en el maxilar superior, y reflejaría una condición ósea del esqueleto y por lo tanto, una posible detección precoz de esta condición, pudiendo diagnosticar un aumento en el riesgo de fractura $(1,4)$. El espesor del mineral óseo 0 densidad mineral ósea (DM0) del hueso mandibular, se relaciona con la DMO de la columna lumbar y del cuello del fémur, áreas más propensas a osteoporosis (20). Para valorar la calidad y cantidad del hueso mandibular, las radiografías panorámicas son las más recomendadas, por considerarse de mayor uso como exámenes de rutina en la salud bucal (21). Es así como se han propuesto índices para poder evaluar la cantidad y calidad del hueso cortical mandibular en las radiografías panorámicas y poder diferenciar entre pacientes con riesgo 0 no de osteoporosis (20), como el índice cortical mandibular (ICM) propuesto por Klemetti y col. $(22,23)$. Otros medios de diagnóstico incluyen la tomografía de haz cónico (23) y la densitometría ósea, que emplea la absorciometría dual de Rayos $X$ de doble energía (DEXA-DXA), considerada una técnica superior y concluyente de osteoporosis $(24,25)$.

\section{ETIOLOGÍA Y FACTORES DE RIESGO} DE OSTEOPOROSIS

La osteoporosis, de las raíces griegas para "hueso" y "poro", es una enfermedad sistémica del componente óseo del cuerpo humano, que presenta una disminución de la DM0, deterioro en la microestructura ósea con adelgazamiento de las trabéculas, cortical ósea y aumento de los espacios medulares, lo que provoca que los huesos sean más frágiles y susceptibles a fracturas (26-28).

Los factores de riesgo para la osteoporosis han sido clasificados como no modificables y modificables (8). Los primeros incluyen la edad, sexo, factores genéticos y menopausia temprana; mientras que entre los segundos se consideran un inadecuado consumo de calcio, la falta de ejercicio, el alcoholismo, el tabaquismo, ciertas enfermedades endocrinas como el hiperparatiroidismo, la insuficiencia renal crónica, la enfermedad hepática, la malabsorción y fármacos como los glucocorticoides orales o los inhibidores de la bomba de protones (reguladores de la acidez) $(8,9)$. La diminución de estrógenos y calcio en mujeres posmenopáusicas se consideran como los principales factores de la osteoporosis (10-12). Entre otros factores de riesgo asociado a osteoporosis se consideran los niveles altos de homocisteína y bajos niveles de lipoproteína de alta densidad (HDL) o colesterol bueno. La homocisteína se relaciona con la enfermedad cardiovascular y puede aumentar con la edad en mujeres posmenopáusicas $(13,14)$. Los factores metabólicos asociados o no con hemodiálisis y la paratohormona, factores genéticos y ambientales durante el desarrollo fetal en la infancia y la vida adulta, podrían también asociarse con una baja DMO (29). El gen de la osteoprotegerína (OPG), participa en el control de la resorción ósea e 
influenciaría en la etiología de la osteoporosis (30-33). Se ha asociado el gen de OPG con una baja DMO en mujeres posmenopáusicas, sin embargo, estos estudios no han sido del todo concluyentes y se requieren más y mejores investigaciones sobre esta asociación.

La disfunción hepática es la segunda causa de osteoporosis u osteopenia. La osteodistrofia hepática, asociada a una enfermedad hepática crónica, se relaciona con osteopenia, osteoporosis y osteomalacia en pacientes con cirrosis hepática (34). La pérdida de DMO en estos pacientes se ha estimado entre el $12 \%$ al $55 \%(35,36)$. Por lo tanto, antes de realizar una intervención quirúrgica que involucre hueso en pacientes con cirrosis, es recomendable tener los cuidados necesarios y poder detectar a tiempo posibles problemas de osteoporosis. El hígado es un órgano importante relacionado con las actividades metabólicas, interviene en factores generales como el procesamiento de la vitamina $D$ y las alteraciones metabólicas del calcio, el hiperparatiroidismo secundario, la deficiencia de vitamina $\mathrm{K}$, la disfunción hormonal, las citoquinas y la deficiencia del factor de crecimiento tipo insulina 1 (IGF-1) que participa en el metabolismo óseo (34). La insuficiencia hepática crónica, así como otros trastornos sistémicos hematológicos, endocrinos o metabólicos, pueden afectar los tejidos duros y blandos de la cavidad oral (35-38).

\section{RELACIÓN DE OSTEOPOROSIS CON LA CAVIDAD BUCAL}

Según la OMS la osteoporosis y la enfermedad periodontal son dos enfermedades cuya incidencia es mayor a medida que avanza la edad y son consideradas como un problema de salud pública. La osteoporosis puede afectar cualquier tipo de hueso, por lo que también podría observarse disminución de DMO en los huesos maxilares, lo que contribuye a que el hueso de soporte sea susceptible a enfermedad periodontal
$(39,40)$. Actualmente, existen estudios donde se asocia la osteoporosis con problemas de salud bucal, como la enfermedad periodontal (16), densidad de la mandíbula (20-23), disminución de su altura, reducción de la anchura cortical inferior y disfunción de las articulaciones temporomandibulares $(4,16)$. De los estudios que reportan la asociación de osteoporosis con la cavidad bucal, se relacionó principalmente con la enfermedad periodontal y la pérdida ósea por osteoporosis (16). Sin embargo, aún faltan más estudios específicos en pacientes que presenten condiciones normales de salud oral; porque con el avance de la edad surgen otros factores de comorbilidad, que podrían inducir la pérdida de hueso y como consecuencia los dientes. Se necesitarían estudios más concluyentes para poder determinar la relación entre la pérdida ósea sistémica de la cavidad oral con osteoporosis.

\section{MÉTODOS DE DIAGNÓSTICO IMAGENOLÓGICOS ADECUADOS Y COMPLEMENTARIOS PARA EL DIAGNÓSTICO DE OSTEOPOROSIS}

La radiografía panorámica ofrece datos fiables con índices de evaluación para detectar disminución de DMO, principios o presencia de osteopenia u osteoporosis. Muchos pacientes con osteoporosis no presentan síntomas antes de tener una fractura y la radiografía panorámica al ser un examen que se solicitada con relativa frecuencia por los odontólogos, sería considerada un medio ideal de diagnóstico. Se tienen los siguientes índices de evaluación: (i) el índice mandibular panorámico (IMP), (ii) el ancho de la cortical mandibular (ACM) 0 índice mentoniano (IM), son relacionados con la cantidad de hueso o DMO. El (iii) índice de la cortical mandibular (ICM), que se relaciona con la calidad del hueso a través de la forma de la mandíbula $(4,24)$, (iv) el índice gonial $(\mathrm{IG})$ y $(\mathrm{V})$ antegonial $(\mathrm{IA})$, que miden grosor posterior y anterior de la rama mandibular (no son estables) y (vi) la relación mandíbulomandibular $(\mathrm{M} / \mathrm{M})$, que es la relación entre la altura total del cuerpo mandibular y la altura 
desde el borde inferior de la mandíbula al borde inferior del agujero mentoniano, considerándose el menos significativo $(3,41,42)$. El ICM, el ACM ó IM (43) y el IMP serían los más significativos y estables para la predicción y detección precoz de osteoporosis en mujeres posmenopáusicas con baja DM0 $(42,44)$.

Para evaluar la forma de la cortical mandibular, se tiene el ICM donde se observan los cambios producidos desde la cortical mandibular a distal del foramen mentoniano de forma bilateral, de acuerdo a la siguiente escala según Klemetti y colaboradores $(4,22,43)$ (Fig.1).

- Corteza normal (C1): El margen cortical endosteal es uniforme y afilado en ambos lados.

- Corteza leve a moderadamente erosionada (C2): El margen cortical endosteal muestra cavidades de resorción o reabsorción lacunar, con 1 a 2 capas de residuos corticales endosteales en una 0 ambos lados.

- Corteza gravemente erosionada (C3): El margen cortical endosteal, presenta residuos corticales porosos bien marcados.

Para determinar el IMP se mide la distancia entre el borde inferior de la mandíbula y el borde inferior del foramen mentoniano y se divide con el ancho de la cortical mandibular ubicada a nivel del foramen mentoniano. El valor resultante no debe ser inferior a $0.3 \mathrm{~mm}$, lo cual se podría interpretar como una mayor probabilidad de osteoporosis y una baja DMO. El valor del ACM que se mide a nivel del foramen mentoniano, no debería ser menor de $3 \mathrm{~mm}(4,42,46)$ (Fig.2,3). En los estudios del uso de radiografía panorámica para la evaluación de osteoporosis en mujeres posmenopáusicas, el ACM demostró tener una especificidad del 96\% y una sensibilidad del $60 \%$ lo que lo convierte en un indicador eficaz y fiable (4), y a la radiografía panorámica en una herramienta que ofrece buenos parámetros de referencia para la detección precoz de esta enfermedad. Sin embargo, es relevante recalcar que para evaluar las características mencionadas, se requiere de preparación y capacitación adecuada por parte del observador, pudiendo variar de un odontólogo (o radiólogo) a otro, por su experiencia.

El término tomografía computarizada cone beam 0 de haz cónico está formada por la unión de dos letras griegas "tomos" y "graphos" qué significan, "partes" y "registro". Esto implica la obtención de imágenes del cuerpo en partes 0 cortes. En el campo de la odontología, el progreso tecnológico ha sido amplio en lo que se refiere al diagnóstico por imagen en el área digital. Es así que surge la tomografía de haz cónico, presentando ventajas sobre el tomógrafo médico, ya que esta utiliza una menor radiación y el área de estudio es más específica lo que previene distorsiones y ofrece una ubicación exacta del conducto dentario inferior y un registro de partes con reconstrucción volumétrica sin superposiciones de imágenes (47).

El uso de este medio de diagnóstico en la identificación de mujeres posmenopáusicas con osteoporosis, ha demostrado ser de ayuda al evaluar la DMO a través de índices para tomografía computarizada, tales como el índice mandibular de tomografía computarizada superior (ITCS) que es la relación entre el ancho de la cortical mandibular a nivel del foramen mentoniano y la distancia del borde superior del foramen mentoniano al borde inferior mandibular. Además, se ha propuesto el índice mandibular de tomografía computarizada inferior (ITCl), que es la relación entre el ancho de la cortical mandibular a nivel del foramen mentoniano y la distancia del borde inferior del foramen mentoniano al borde inferior mandibular. Estos serían los dos índices más utilizados para diferenciar entre valores normales y valores que impliquen mayor riesgo a osteoporosis, pudiendo relacionarse con el IMP. Mientras que el índice que mide la cortical mandibular en tomografía computarizada (ICTC), resultó ser menos significativo en relación a la radiografía 
panorámica (48) y utiliza datos similares al ICM según Klemetti, pero en el caso del ICTC, la escala seria: tipo 1, 2 y 3 (49).

Estudios que evaluaron la estructura ósea trabecular de los maxilares en TCHC, no revelaron grandes cambios que podrían identificarse como osteoporosis, arrojando datos más significativos de osteoporosis al analizar y evaluar el trabeculado óseo de la $2 \mathrm{da}$ vértebra cervical a nivel del odontoides (50), es así que mediante la tomografía y la resonancia magnética, se podría evaluar la estructura del hueso trabecular, midiendo el volumen, el espaciado y la conectividad, los estudios son prometedores; pero los costos y la complejidad de estos métodos, serían una limitante del uso diario en la práctica clínica $(50,51)$.

La densitometría ósea 0 absorciometría de rayos $X$ de energía dual (DEXA-DXA), es una forma mejorada de tecnología de rayos $X$ y constituye una prueba estándar concluyente, que permite medir la densidad de calcio que existen en los huesos y/0 evaluar la DMO como un predictivo de riesgo de fractura $(25,52-54)$. Esta prueba es más recomendada en mujeres mayores de 65 años y hombres de 70 años o más, así como en personas con riesgo de osteoporosis, como mujeres posmenopáusicas, personas que han sufrido fracturas por traumatismo bajo a los 50 años 0 más, por lo tanto para evaluar un riesgo de fractura clínica, se aconseja realizar exámenes para evaluar la densitometría ósea a partir de los 50 años de edad y en personas que presentan los factores de riesgo anteriormente descritos (53). Si no se dispone de este examen, la obtención de imágenes vertebrales sería una buena opción y debería realizarse en mujeres mayores de 70 años y en hombres mayores de los 80 años, con pérdida histórica de altura de $4 \mathrm{~cm} 0+$ (diferencia entre altura actual y altura máxima a los 20 años) 0 posible pérdida de $2 \mathrm{~cm} 0+$ (diferencia entre altura actual y medición de altura anteriormente documentada) $(53,54)$. Según la
OMS, el diagnóstico de osteoporosis con DXA debería ser de preferencia en columna lumbar y cadera; y sugiere un seguimiento cada 1 a 2 años de acuerdo a la gravedad de dicha enfermedad y la verificación de la efectividad del tratamiento instaurado mediante una DXA central (55). La DXA es una técnica radiográfica costosa, que implica mayor radiación y no es considerada como un examen de rutina en detección de osteoporosis en mujeres posmenopáusicas $(56,57)$.

Dentro los estudios complementarios de laboratorio para diagnosticar osteoporosis, existen marcadores bioquímicos como la osteocalcina sérica, la fosfatasa alcalina total sérica y la hidroxiprolina en orina, los cuales indican el estado del recambio óseo en todo el sistema esquelética en general; pero no ofrece valores para zonas específicas (1). La osteocalcina, un marcador de formación ósea generada por osteoblastos, puede impedir la síntesis de colágeno, generando la reabsorción de hueso (58). Se han encontrado elevados niveles de osteocalcina en casos como osteoporosis y reparación de fracturas, donde existe una rápida pérdida ósea (59).

El calcio, es un elemento químico abundante en el organismo y casi el $98 \%$ de calcio del cuerpo se encuentra depositado en el tejido óseo y lo restante se encuentra en los líquidos corporales. Este, desempeña un papel biológico muy importante ya que es un cofactor enzimático en muchos procesos biológicos y actividades hormonales para el organismo. La cantidad de calcio presente en la sangre llamada calcemia, es regulada por la actividad de la hormona paratiroidea (PTH) y la vitamina D. Cuando disminuye el calcio ionizado, la acción determinada de la PTH y la vitamina D incrementan la absorción de calcio a nivel intestinal, la liberación de calcio del esqueleto y la conservación del mineral a nivel renal (60). La tabla 1 resume los valores normales de concentración plasmática de calcio sérico y calcio iónico (60). Los niveles de fósforo sérico no son tan rígidos, en 
relación a las concentraciones de calcio, son más flexibles de acuerdo al aporte de fósforo contenido en la dieta. El 85\% circula libre en la sangre y tiene ligera relación con las proteínas plasmáticas. Las concentraciones de fósforo en sangre son más altas en los primeros años de vida y luego disminuye con la edad. El índice calcio urinario/ creatinina, nos permite valorar de forma indirecta el manejo renal de calcio, y se constituye en una gran ayuda para hacer el seguimiento de la respuesta al tratamiento de enfermedades y problemas de hipocalcemia (60). La tabla 1 resume los valores normales de estos marcadores bioquímicos. Los estudios de laboratorio solicitados deberían incluir los niveles de calcio y creatinina, debido a la importancia del nivel de calcio en sangre y la función renal, complementando con un examen completo de hemograma (55).
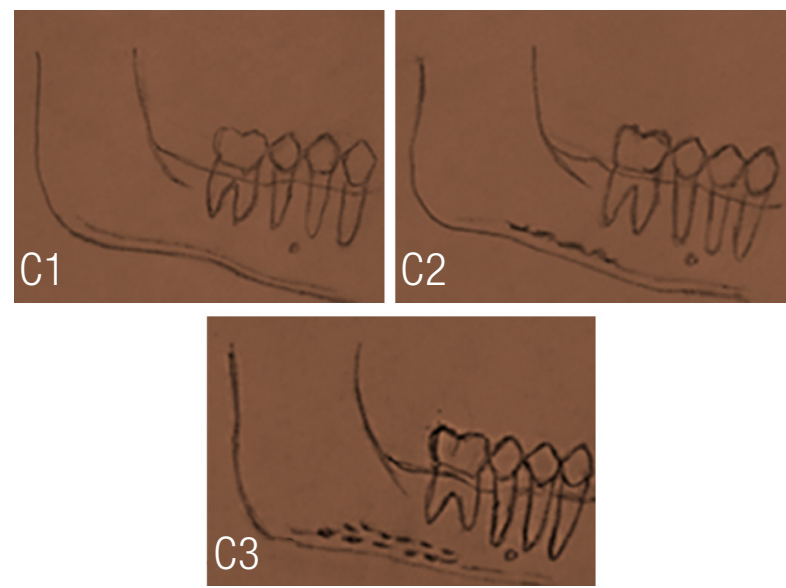

Fig. 1. Esquema gráfico del índice de la cortical mandibular (ICM) C1 normal, cortical endosteal uniforme y afilado en ambos lados, C2 leve o moderado, cortical endosteal con cavidades de resorción, C3 grave, cortical endosteal con residuos corticales porosos bien marcados. Fuente: elaboración propia.

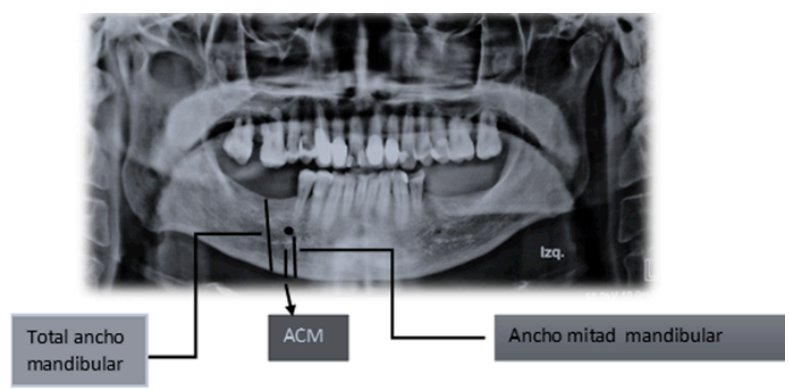

Fig. 2. Esquema que muestra el ancho cortical mandibular (ACM) El ancho mitad mandibular, va del agujero mentoniano a la base de la cortical mandibular. Total ancho mandibular que va del reborde alveolar a la base de la cortical mandibular. Fuente: elaboración propia.

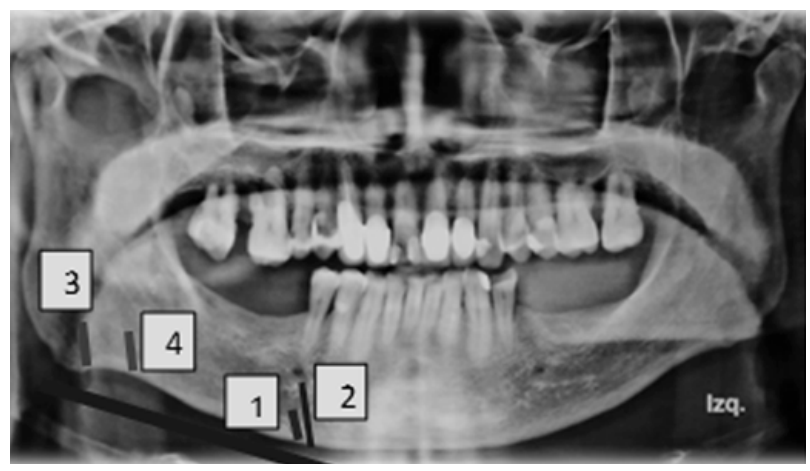

Fig. 3. Ancho cortical mandibular (ACM) 1, índice mandibular panorámico (IMP) es la diferencia entre 2 y 1 , índice gonial (Gl) 3, índice antegonial (Al) 4, donde 3 y 4 evalúan el grosor mandibular en la parte posterior y anterior de la rama. Fuente: elaboración propia.

Tabla 1. Valores normales en adultos de la concentración plasmática de los marcadores bioquímicos calcio, fósforo y creatinina.

\begin{tabular}{ll}
\hline & Valores normales en adultos \\
\hline Calcio Sérico & $8,8-10,4 \mathrm{mg} / \mathrm{dl}(2,2-2,6 \mathrm{mmol} / \mathrm{l})$ \\
Calcio lónico & $4,4-5,2 \mathrm{mg} / \mathrm{dl}(1,1-1,3 \mathrm{mmol} / \mathrm{l})$ \\
Fósforo Sérico & $3,5 \mathrm{mg} / \mathrm{dl}(1,1 \mathrm{mmol} / \mathrm{l})$ \\
Creatinina & Hombres: 0.7 a $1.3 \mathrm{mg} / \mathrm{dl}(61,9$ a 114,9 \\
& $\mathrm{mmol} / \mathrm{l})$ \\
& Mujeres: 0.6 a $1.1 \mathrm{mg} / \mathrm{dl}(53$ a $97.2 \mathrm{mmol} / \mathrm{l})$ \\
\hline
\end{tabular}




\section{CONCLUSIÓN}

De acuerdo con la revisión de literatura realizada, la detección de osteoporosis en los maxilares mediante exámenes imagenológicos, mostró que el uso de la radiografía panorámica, mediante los índices radiográficos, ofrecen una adecuada valoración de la densidad mineral ósea y riesgo a padecer osteoporosis a través de datos simples y menos costosos en relación a un examen tomográfico, que implicaría un mayor costo y de menor accesibilidad para la población en general. El examen de densitometría ósea, si bien es un estudio concluyente, no es considerado de uso rutinario para detectar osteoporosis, ya sea por su costo o por la mayor exposición a radiación en relación a una radiografía panorámica. Se aconseja utilizarlo solo después de obtener evidencia clínica e índices radiográficos que indiquen una mayor probabilidad de padecer esta enfermedad 0 en pacientes con riesgo aumentado a padecerla. La confirmación de osteoporosis se da con la densitometría ósea sumada a la evaluación de marcadores bioquímicos que mejoran la predicción, diagnóstico y clasificación del grado de severidad de osteoporosis.

\section{REFERENCIAS}

1. Jonasson G., Skoglund I., Rythen M. The rise and fall of the alveolar process:Dependency of teeth and metabolic aspects. Arch Oral Biol 2018; 96: 195-200.

2. Rachner T. D., Khosla S., Hofbauer L. C. Osteoporosis: now and the future. Lancet 2011; 377 (9773): 1276-87.

3. Calciolari E., Donos N., Park J. C., Petrie A., Mardas N. Panoramic measures for oral bone mass in detecting osteoporosis: a systematic review and meta-analysis. J Dent Res 2015; 94 (3) 17-27.

4. Bhatnagar S., Krishnamurthy V., Pagare S. S. Diagnostic efficacy of panoramic radiography in detection of osteoporosis in post-menopausal women with low bone mineral density. J Clin Imaging Sci 2013; 3: 23.

5. Yasar F., Akgunlu F. Evaluating Mandibular Cortical Index Quantitatively. Eur J Dent. 2008; 2: 283-90.

6. Mendoza N., Sanchez-Borrego R., Villero J., et al. Up-date of the consensus statement of the Spanish Menopause Society on postmenopausal osteoporosis. Maturitas 2013; 76 (1): 99-107.

7. Pinheiro M., Reis E., Machado F., et al. Risk factors for osteoporotic fractures and low bone density in pre and postmenopausal women. Rev. Saúde Pública 2010; 44 (3): 479-85.

8. Coat J. The association between osteoporosis and oral bone loss. J Periodontology 2005; 76 (11): 2125-32.

9. Cole Z., Dennison E., Cooper C. The impact of methods for estimating bone health and the global burden of bone disease. Salud Pública Mexico 2009; 51 (1): 38-45.

10. Raisz L. G. Pathogenesis of osteoporosis: concepts, conflicts, and prospects. J Clin Invest 2005; 115 (12): 3318-25.

11. Dick I. M., Devine A., Beilby J., Prince R. L. Effects of endogenous estrogen on renal calcium and phosphate handling in elderly women. Am J Physiology Endocrinology and Metabolism 2005; 288 (2): 430-35.

12. Meng J., Zhang D., Pan N, et al. Identification of miR-194-5p as a potential biomarker for postmenopausal osteoporosis. Peer J 2015; 3: 971.

13. Saoji R., Das R. S., Desai M., et al. Association of high-density lipoprotein, triglycerides, and homocysteine with bone mineral density in young Indian tribal women. Arch Osteoporos 2018; 13 (1): 108.

14. Farhat G., Cauley J. The link between osteoporosis and cardiovascular disease. Clin Cases Miner Bone Metab 2008; 5 (1): 19-34. 
15. Bone S. Clinician's Guide to Prevention and Treatment of Osteoporosis. National Osteoporosis Fundation 2008; 25 (10): 2359-81.

16. Emel D. Oral implications of osteoporosis. Oral Surg, Oral Med, Oral Pathol,Oral Radiol and Endod 2005; 100 (3): 349-56.

17. Gur A., Nas K., Kayhan O. The relation between tooth loss and bone mass in postmenopausal osteoporotic women in Turkey. J Bone Miner Metab 2003; 21 (1): 43-47.

18. Moedano D. E., Irigoyen M. E., Borges Y. A. Osteoporosis, the risk of vertebral fracture, and periodontal disease in an elderly group in Mexico City. Gerodontology 2011; 28: 19-27.

19. Yoshihara A., Seida Y., Hanada N. The relationship between bone mineral density and the number of remaining teeth in communitydwelling older adults. J Oral Rehabil 2005; 32: 735-40.

20. MarandiS., BagherpourA.,Imanimoghaddam M., Hatef M., Haghighi A. Panoramic based mandibular indices and bone mineral density of femoral neck and lumbar vertebrae in women. J Dent (Tehran). 2010; 7 (2): 98-106.

21. Cakur B., Dagistan S., Sahin A., Harorli A., Yilmaz A. B. Reliability of mandibular cortical index and mandibular bone mineral density in the detection of osteoporotic women. Dentomaxillofac Radiol 2009; 38: 255.

22. Quevedo P., Hernández A. Evaluación de la densidad mineral ósea mandibular a través de la radiografía panorámica.Assessment of mandibular bone mineral density through panoramic radiography. Odous Cientifica 2011; 12 (2): 22-30.

23. Kato C. N., Tavares N. P., Barra S. G., et al. Digital panoramic radiography and cone-beam CT as ancillary tools to detect low bone mineral density in post-menopausal women. Dentomaxillofac Radiol 2019; 48 (2): 20180254.
24. Taguchi A., Sugino N., Miki M., et al. Triage screening for osteoporosis in dental clinics using panoramic radiographs. Dentomaxillofacial Radiology 2010; 16 (4): 316-27.

25. Güngör E., Yildirim D., Çevik R., et al. Evaluation of osteoporosis in jaw bones using cone beam $\mathrm{CT}$ and dual-energy X-ray absorptiometry. J Oral Sci 2016 2016; 58: 185-94.

26. Lorentzon M., Cummings S. R. Osteoporosis: the evolution of a diagnosis. J Intern Med 2015; 277 (6): 650-61.

27. Cummings S. R., Melton L. J. Epidemiology and outcomes of osteoporotic fractures. Lancet 2002; 359 (9319): 1761-67.

28. Piotr G., Roman S., Lorenc., et al. Polish guidelines for the diagnosis and management of osteoporosis. Pol Arch Med Wewn 2013; 124: 255-63.

29. Binici D. N., Gunes N. Risk factors leading to reduced bone mineral density in hemodialysis patients with metabolic syndrome. Ren Fail 2010; 32: 469-74.

30. Shouyi L., Huiqiang J., Ningke D. Association Analysis between g.18873C $>$ T and g. $27522 \mathrm{G}>\mathrm{A}$ genetic polymorphisms of OPG and bone mineral density in Chinese postmenopausal women. Biomed Res 2017; 12 (12).

31. Mydlárová B. M., Blaščáková L., Poráčová J., et al. Relationship between A163G osteoprotegerin gene polymorphism and other osteoporosis parameters in Roma and non-Roma postmenopausal women in eastern Slovakia. J Clin Lab Anal 2017; 31 (5).

32. Hofbauer L. C., Khosla S., Dunstan C. R., et al. The roles of osteoprotegerin and osteoprotegerin ligand in the paracrine regulation of bone resorption. . J Bone Miner Res 2010; 15 (1): 2-12. 
33. Xiaoyong S., Guangyong C., Xingjun G., Zouying Y., Ye Z. Common variants in $\mathrm{OPG}$ confer risk to bone mineral density variation and osteoporosis fractures. Sci Rep 2017; 7: 1739.

34. Handzlik-Orlik G., Holecki M., Wilczyński K., Duława J. Osteoporosis in liver disease: pathogenesis and management. Therapeutic Advances in Endocrinology and Metabolism 2016; 7 (3): 128-35.

35. Karoli Y., Karoli R., Fatima J., Manhar M. Study of hepatic osteodystrophy in patients with chronic liver disease. Journal of Clinical and Diagnostic Research 2016; 10 (8): 31-34.

36. Gatta A., Verardo A., Di Pascoli M., Giannini S., Bolognesi M. Hepatic osteodystrophy. Clinical Cases in Mineral and Bone Metabolism 2014; 11 (3): 185-91.

37. Helenius-Hietala J., Ruokonen H., Grönroos L., etal. Oral mucosal health in liver transplant recipients and controls. Liver Transplantation 2014; 20 (1): 72-80.

38. Ghapanchi J., Rezaee M., Kamali F., Lavaee F., Shakib E. Prevalence of oral and craniofacial manifestations of hematological dyscrasias at Shiraz Nemazee Hospital. Middle East Journal of Cancer. 2014; 5 (3): 145-49.

39. Velásquez G. Prevalencia de la pérdida dentaria en pacientes con osteoporosis. Odous Cientifica 2009; 10 (2): 16-21.

40. Hildebold C. Osteoporosis and oral bone loss. Dentomaxillofacial Radiology 1997; 26 (3): 15.

41. Dutra V., Devlin H., Susin C. Cambios morfológicos mandibulares en mujeres edéntulas con baja masa ósea: evaluación de radiografías panorámicas. Cirugía oral Oral Med. Oral Pathol Oral Radiol Endod 2006; 102: 663-68.

42. Corcuera Flores J. R., Manso Platero F. J., Martínez Maestre M. A., Machuca Portillo G. Actualizaciones en la detección precoz de la osteoporosis. Av odontoestomatol 2014; 30 (5).

43. Devlin H., Horner K. Indices radiomorfométricos mandibulares en el diagnóstico de densidad mineral ósea esquelética reducida. Osteoporos Int 2002; 13: 373-78.

44. Pavicin I. S., Dumancic J., Jukic T. Ortopantomogramas digitales en la detección de osteoporosis: densidad mandibular e índices radiográficos mandibulares como predictores de la DMO esquelética. Dentomaxillofac 2014; 43.

45. Klemetti E., Kolmakov S., Heiskanen P., Vainio P., Lassila V. Panoramic mandibular index and bone mineral densities in postmenopausal women. Oral Surg Oral Med Oral Pathol 1993; 75 (6): 774-79.

46. Taguchi A., Ohtsuka M., Tsuda M., et al. Riesgo de osteoporosis vertebral en mujeres posmenopáusicas con alteraciones de la mandíbula. Dentomaxillofac Radiol 2007; 36: 143-48.

47. Santana S. T., Carvalho R. R., Rocio M. A., et al. El uso de la tomografia computarizada de haz volumetrico en odontologia. Odontol Clin Cient 2010; 9 (4): 303-06.

48. Koh K. J., Kim K. A. Utility of the computed tomography indices on cone beam computed tomography images in the diagnosis of osteoporosis in women. Imaging Sci Dent 2011; 41 (3):101-06.

49. Mostafa R. A., Arnout E. A., Abo El Fotouh M. M. Viabilidad del análisis radiomorfométrico y la dimensión fractal de la tomografía computarizada de haz cónico en la evaluación de la osteoporosis posmenopáusica en correlación con la absorciometría dual de rayos X. Dentomaxillofac Radiol 2016; 45 (7).

50. Barngkgei I., Al Haffar I., Shaarani E., Khattab R., Mashlah A. Assessment of jawbone trabecular bone structure amongst 
osteoporotic women by cone-beam computed tomography: the OSTEOSYR project. J Investig Clin Dent. 2016; 7 (4): 332-40.

51. Brasileiro C. B., Chalub L. L. F. H., Abreu M. H. N. G., et al. Use of cone beam computed tomography in identifying postmenopausal women with osteoporosis. Archivos de osteoporosis 2017; 12 (1): 26.

52. Kazakia G. J., Majumdar S. New imaging technologies in the diagnosis of osteoporosis. Rev Endocr Metab Disord 2006; 7 (67-74).

53. Cosman F., De Beur S. J., LeBoff M. S., et al. Errata a: Clinician's guide to prevention and treatment of osteoporosis. Osteoporos Int 2015; 26 (7): 2045-47.

54. Group Roa W. S. Assessment of fracture risk and its application to screening for postmenopausal osteoporosis. World Health Organ Tech Rep Ser 1994; 843: 1-129.

55. Camacho P. M., Petak S. M., Binkley N. Guía de práctica clínica para el diagnóstico y tratamiento de con la osteoporosis posmenopáusica. Endocr Pract 2016; 22 1-42.
56. Balto K. A., Gomaa M. M., Feteih R. M., et al. Dental Panoramic Radiographic Indices as a Predictor of Osteoporosis in Postmenopausal Saudi Women. J Bone Metab 2018; 25 (3): 165-73.

57. Nakamoto T., Taguchi A., Ohtsuka M. Dental radiography as a tool to detect postmenopausal women with low bone mineral density: diagnostic performance of general dentists without training. Osteoporos 2003; 14: 659-64.

58. Lian J. B., Gundberg C. M. Osteocalcin. Biochemical considerations and clinical applications. Clin Orthop Relat Res 1988; 226 (226): 227-91.

59. YamazuraS.,AloiaJ. Serumosteocalcinandtotal bodycalcium innormal, preand postmenopausal women and post-menopausalosteoporotic patients. J Clinic End Met 1987; 64: 681.

60. Yeste D., Carrascosa A. Patología del metabolismodelcalcio. Protocolos actualizados, Servicio de Endocrinología Pediátrica. Hospital Materno-infantil Vall d'Hebron. Barcelona 2011; 1: 177-92. 